\title{
Correction to: A Container-Oriented Virtual- Machine-Introspection-Based Security Monitor to Secure Containers in Cloud Computing
}

Zhaofeng Yu(D, Lin Ye, Hongli Zhang, Dongyang Zhan, Shen Su, and Zhihong Tian

Correction to:

Chapter "A Container-Oriented Virtual-MachineIntrospection-Based Security Monitor to Secure Containers in Cloud Computing" in: X. Sun et al. (Eds.): Artificial Intelligence and Security, LNCS 12737, https://doi.org/10.1007/978-3-030-78612-0_8

The original version of this chapter was revised. Shen Su and Zhihong Tian have been added as co-authors and their affiliation have been added, as they made important contributions to the paper. 